\title{
Palmprint recognition using multiscale transform, linear discriminate analysis, and neural network
}

\author{
Hatem Elaydi ${ }^{1}$, Mohanad A. M. Abukmeil ${ }^{1}$, Mohammed Alhanjouri ${ }^{2}$ \\ ${ }^{1}$ Electrical Engineering, the Islamic University, Gaza, Palestine \\ ${ }^{2}$ Computer Engineering, the Islamic University, Gaza, Palestine
}

\section{Email address:}

helaydi@iugaza.edu.ps(H. Elaydi),mohanadxyz@outlook.com(M. A. M. Abukmeil)

\section{To cite this article:}

Hatem Elaydi, Mohanad A. M. Abukmeil, Mohammed Alhanjouri. Palmprint Recognition Using Multiscale Transform, Linear Discriminate Analysis, and Neural Network. Science Journal of Circuits, Systems and Signal Processing. Vol. 2, No. 5, 2013, pp. 112-118. doi: 10.11648/j.cssp.20130205.13

\begin{abstract}
Palmprint recognition is gaining grounds as a biometric system for forensic and commercial applications. Palmprint recognition addressed the recognition issue using low and high resolution images. This paper uses PolyU hyperspectral palmprint database, and applies back-propagation neural network for recognition, linear discriminate analysis for dimensionality reduction, and 2D discrete wavelet, ridgelet, curvelet, and contourlet for feature extraction. The recognition rate accuracy shows that contourlet outperforms other transforms.
\end{abstract}

Keywords: 2D Discrete Wavelet, Ridgelet, Curvelet, Contourlet, Linear Discriminate Analysis, Neural Network

\section{Introduction}

A biometric system may be used for personal identification instead of token-based methods such as a passport, a physical key and an ID card or Knowledgebased method such as a password. In the token-based, "token" can be stolen or lost easily while knowledge can be forgotten or guessed in a knowledge-base [1].

Palmprint identification has emerged as one of the leading and promising biometric modalities for forensic and commercial applications [2.3]. Palmprint features are considered unique and have a real potential in identify people. Palmprint features can be classified, with reference to the field at which palmprint systems are used, into two groups. The first group of features are the principal lines and wrinkles which could be extracted from low resolution images $(<100 \mathrm{dpi})$ and may be used in the commercial applications. The second group of features are the singular point, ridges and minutiae point which could be extracted from high resolution images (>100dpi) and may be used for forensic applications such as law enforcement application [3]. Both high and low resolution image features in palmprint are shown in Fig. 1.

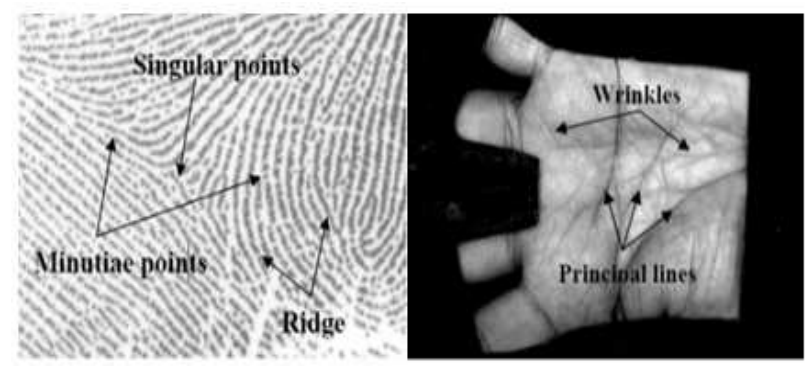

Fig 1. Palmprint features

This paper uses multiscale image transforms such as $2 \mathrm{D}$ discrete wavelet, ridgelet, curvelet and contourlet for feature extractions from palmprint images, and compares their results. It also uses $2 \mathrm{D}$ linear discriminate analysis for dimensionality reduction and back- propagation neural network for recognition.

The rest of this paper is organized as follows: Section 2 gives a brief description of related work. Multiscale image transform, dimensionality reduction 2D LDA, in addition to feed-forward back-propagation neural network will be highlighted in section 3. Section 4 reports feature extraction and recognition results for each multiscale image transform. Finally, the conclusion and future work are presented in section 5 . 


\section{Related Work}

Many researchers have been investigating the identification of people using palmprint features. Various techniques have gained popularity to extract features from palmprint images.

Kekre, et.al. (2012) [4], suggested the use of a hybrid wavelet generated by Kronecker product of two existing orthogonal transforms, Walsh and DCT, to identify multispectral palmprints. One-to-many identification on a large database containing three sets of 6000 multi-spectral palmprint images from 500 different palms was used to validate the performance. The matching accuracy of the proposed method of genuine acceptance ratio of $99.979 \%$ using score level fusion was obtained. Selection feature vectors depended on high energy components and was insufficient to select the most discriminative feature. However, the recognition phase was complex and time consuming.

Sharkas, et.al. (2010) [5], compared two techniques for palmprint recognition. The first technique extracted the edges from the palm images, then performed the CT or the Discrete Wavelet Transform (DWT) on the edge extracted images. The second technique employed the principal component analysis PCA. Features extracted from both techniques were tested and compared where it was found that the best achieved recognition rate was about $94 \%$. However, ROI in this paper was not clear; the minimum distance classifier used was insensitive to differences in variance. Five palmprint images were trained and the recognition depended on the number of eigenvectors which was insufficient.

Masood, et.al. (2009) [6], suggested a palmprint based identification approach that drew on the textural information available on the palmprint by utilizing a combination of contourlet and non-subsampled contourlet transforms. The algorithm was tested on a 500 palm images of GPDS hand database. The results of the proposed algorithm were compared with reported results in literature. The proposed algorithm outperformed other reported methods of palmprint matching using equal error rate (EER). ROI was $256^{\times} 256$ pixels which may increase the complexity in some phases. The selected features may be inadequate to distinguish the different classes. The selected features could also be highly correlated and features space may simply be too complex with the limitations of Euclidean distance classifier.

Jiwen, et.al. (2006) [7], proposed using wavelet decomposition and 2D principal component analysis (2DPCA) for palmprint recognition. 2D wavelet transform and 2DPCA were applied to the low-frequency components. The algorithm used the Poly palmprint image database and the experimental results were encouraging and achieved comparatively high recognition. The major limitation consists of using only 100 palmprints and six samples for each palm. In addition, the number of training and testing palms where inconsistent. Another limitation in comparison with other projection techniques, the comparison was done with PCA and ICA while used images require 2-D domain. Ten projection vectors were used as classifier input resulting in time consuming and high complexity.

The major disadvantages of presented work are the high implementation complexity, execution time, cost, etc. The classifier type in some researches may be time consuming and has reliability issue when it compared with neural network classifier. The number of vectors that used as a classifier input in some researches is more than one vector meaning that recognition may consume more time. The projection technique may not supports $2 \mathrm{D}$ domain and the combination between the classifier and image transform technique may be inconsistent. In order to overcome the disadvantages of existing techniques, a new palmprint recognition based on the combinations between multiscale image transforms, dimensionality reduction by 2D LDA and back-propagations neural networks that require less formal statistical training for recognition was proposed.

\section{Multiscale and Classifier}

Multiscale describes a passband system with a spatial scale controlled by a single parameter such as linear filters with the wavelength as a parameter. In this case, the wavelength is closely related to resolution such that short wavelengths are needed to describe small sized objects associated with fine resolution [7].

\subsection{Transforms}

\subsubsection{The 2D Discrete Wavelet}

The 2D DWT $[8,9]$ is used in compression, denoising and watermarking applications. It is built with separable orthogonal mother wavelets $(\psi)$ with a given regularity. At every iteration of the DWT, the lines of the input image (obtained at the end of the previous iteration) are low-pass filtered with a filter having the impulse response $m_{0}$ and high-pass filtered with the filter $m_{1}$. Then, the lines of the two images obtained at the output of the two filters are decimated with a factor of 2 . Next, the columns of the two images obtained are low- pass filtered with $\mathrm{m}_{0}$ and highpass filtered with $m_{1}$. The columns of those four images are also decimated with a factor of 2 .

Four new sub-images (representing the result of the current iteration) are generated. The first one is obtained after two low-pass filtering; it is named approximation subimage (or LL image), the others three are named detail subimages: LH, HL and HH. The LL image represents the input for the next iteration. In the following, the coefficients of the DWT will be noted with $\mathrm{xD}_{\mathrm{m}}^{\mathrm{k}}$ where $\mathrm{x}$ represents the image who's DWT is computed, $\mathrm{m}$ represents the iteration index (the resolution level) and $\mathrm{k}=$ $1,2,3$ and 4 where $\mathrm{k}=1$, for the $\mathrm{HH}$ image, $\mathrm{k}=2$, for the $\mathrm{HL}$ image, $\mathrm{k}=3$, for $\mathrm{LH}$ image and $\mathrm{k}=4$ for the LL image. These coefficients are computed using the following relation: 


$$
x D_{m}^{k}[n, p]=\left\langle x\left(\tau_{1} \tau_{2}\right), \psi_{m, n, p}^{k}\left(\tau_{1}, \tau_{2}\right)\right\rangle
$$

Where the wavelets can be factorized:

$$
\psi_{\mathrm{m}, \mathrm{n}, \mathrm{p}}^{\mathrm{k}}\left(\tau_{1}, \tau_{2}\right)=\alpha_{\mathrm{m}, \mathrm{n}, \mathrm{p}}^{\mathrm{k}}\left(\tau_{1}\right) \cdot \beta_{\mathrm{m}, \mathrm{n}, \mathrm{p}}^{\mathrm{k}}\left(\tau_{2}\right)
$$

And the two factors can be computed using the scale function $\varphi(\tau)$ and the mother wavelets $\psi(\tau)$.

\subsubsection{Continuous Ridgelet Transform}

Given an integrable bivariate function $\mathrm{f}(\mathrm{x})$, its Continuous Ridgelet Transform (CRT) in $\mathbb{R}^{2}$ is defined by $[10,11]$.

$$
\operatorname{CRT}_{\mathrm{f}}(\mathrm{a}, \mathrm{b}, \theta)=\int_{\mathbb{R}^{2}} \psi_{\mathrm{a}, \mathrm{b}, \theta}(\mathrm{x}) \mathrm{f}(\mathrm{x}) \mathrm{dx},
$$

where the ridgelets, $\psi_{\mathrm{a}, \mathrm{b}, \theta}(\mathrm{x})$ in 2-D are defined from a wavelet type function in 1-D $\psi(\mathrm{x})$ as

$$
\Psi_{\mathrm{a}, \mathrm{b}, \theta}(\mathrm{x})=\mathrm{a}^{-\frac{1}{2}} \psi\left(\left(\mathrm{x}_{1} \cos \theta+\mathrm{x}_{2} \sin \theta-\mathrm{b}\right) / \mathrm{a}\right)
$$

Fig. 2 shows an example of ridgelet function, which is oriented at an angle $\theta$ and is constant along the lines

$$
\mathrm{x}_{1} \cos \theta+\mathrm{x}_{2} \sin \theta=\mathrm{const}
$$

The CRT is similar to the 2-D continuous wavelet transform except that the point parameters $\left(b_{1}, b_{2}\right)$ are replaced by the line parameters $(b, \theta)$. In other words, these 2-D multiscale transform are related by:

$$
\begin{aligned}
& \text { Wavelet: } \rightarrow \Psi_{\text {Scale,point_position, }} \\
& \text { Ridgelet: } \rightarrow \psi_{\text {Scale,line_position, }}
\end{aligned}
$$

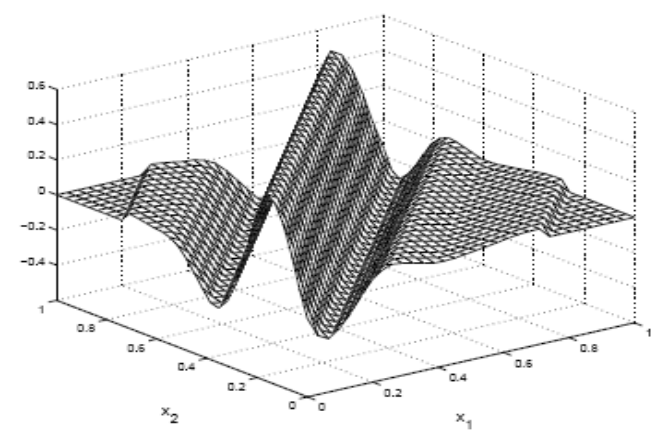

Fig 2. Ridgelet function

As a consequence, wavelets are very effective in representing objects with isolated point singularities, while ridgelets are very effective in representing objects with singularities along lines such as one can think of ridgelets as a way of concatenating 1-D wavelets along lines. Hence, the motivation for using ridgelets in image processing tasks is appealing since singularities are often joined together along edges or contours in images $[12,13]$.

\subsubsection{Curvelet}

Curvelet [14] approximation decomposes the image into a fixed system of components, prescribed without prior knowledge of the image. The curvelet is a family of functions $\gamma_{\mathrm{j}, \mathrm{l}, \mathrm{k}}$ indexed by a scale parameter $\mathrm{j}$, an orientation parameter $\mathrm{l}$ and a position parameter $\mathrm{k} \in \mathbb{R}$, yielding a normalized tight frame of the image space such that for all $\mathrm{f} \in \mathrm{L}^{2}\left(\mathbb{R}^{2}\right)$

$$
\|\mathrm{f}\|_{2}^{2}=\sum_{\mathrm{j}, \mathrm{k}, \mathrm{l}}\left|<f, \gamma_{\mathrm{j}, \mathrm{k}, \mathrm{l}}>\right|^{2}
$$

Using curvelet, image approximation is performed by expanding the input into the curvelet frame and quantizing the coefficients, just as in the wavelet setting. However, the effectiveness of the approximation scheme critically depends on the type of scaling, and the sampling of the various parameters. A comparison of the two types of curvelets is contained in [15].

Curvelet construction corresponds to a critically sampled, multiscale directional filterbank, with angular resolution behaving like $1 / \sqrt{\text { Scale. }}$

In order to ensure that the resulting curvelets are rapidly decreasing, two window functions have been fixed

$$
\mathrm{v}:[-\pi, \pi] \rightarrow \mathbb{C}, \quad \mathrm{w}: \mathbb{R}^{+} \rightarrow \mathbb{C}
$$

Both $\mathrm{v}$ and $\mathrm{w}$ are assumed smooth in addition, for $\mathrm{v}$ we require that its $2 \pi$ periodic extension, $v_{\text {per }}$, is smooth as well. In addition, $\mathrm{w}$ picked to be compactly supported. $\mathrm{v}$ act as angular window; in order to guarantee that the functions constructed from $\mathrm{v}$ are even.

\subsubsection{Digital Curvelet: Contourlet}

The curvelet construction [14] relies on features that are hard to transfer to the discrete setting, such as polar coordinates and rotation where several approaches to were developed, see $[16,17,18]$. The approach introduced by Do and Vetterli [18] is based on fast filterbank algorithms with perfect reconstruction where the tight frame property of curvelets is fully retained, in an algorithmically efficient manner. Moreover, the redundancy of the transform is 1.333 , which is by far better than the factor $6 j+1(j=$ number of dyadic scales in the decomposition) reported in [16].

The discrete implementation follows an analogous structure as shown in Fig.3.

1. The image is passed through a pyramid filterbank, yielding a sequence of bandpassed and subsampled images.

2. Directional filterbanks $[17,19]$, are applied to the difference images in the pyramid, yielding directionally filtered and critically subsampled difference images.

3. The directional filterbanks have an inherent subsampling scheme that makes them orthogonal when employed with perfect reconstruction filters. 


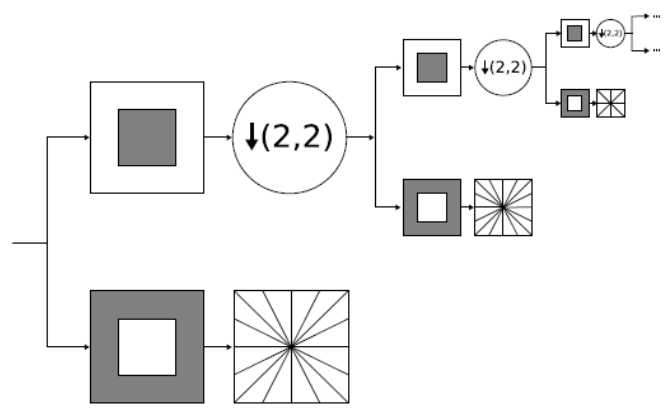

Fig 3. Contourlet decomposition structure

The filterbank uses time-domain filtering, leading to linear complexity decomposition and reconstruction algorithms. In the curvelet/contourlet case, the anisotropic scaling amounts to increasing the angular resolution for large frequencies, which cannot be carried out indefinitely for the discrete domain.

\subsection{The 2D Linear Discriminate Analysis}

Linear discriminate analysis is based on linear combinations between vectors.

2D LDA [22] directly performs discriminate feature analysis on an image matrix rather than on a vector. $2 \mathrm{D}$ LDA tries to find the optimal vector $\mathrm{W}_{\mathrm{opt}}^{2 \mathrm{~d}}$

$$
\mathrm{W}_{\mathrm{opt}}^{2 \mathrm{~d}}=\operatorname{argmax}_{\mathrm{W}^{2 \mathrm{~d}}} \frac{\mathrm{W}^{2 \mathrm{~d}^{\mathrm{T}}} \mathrm{s}_{\mathrm{b}}^{2 \mathrm{~d}} \mathrm{~W}^{2 \mathrm{~d}}}{\mathrm{~W}^{2 \mathrm{~d}^{\mathrm{T}}} \mathrm{s}_{\mathrm{w}}^{2 \mathrm{~d}} \mathrm{~W}^{2 \mathrm{~d}}}
$$

Where $\mathrm{S}_{\mathrm{b}}^{2 \mathrm{~d}}=\sum_{\mathrm{k}=1}^{\mathrm{L}} \frac{\mathrm{N}_{\mathrm{K}}}{\mathrm{N}}\left(\mathrm{U}_{\mathrm{K}}-\mathrm{U}\right)\left(\mathrm{U}_{\mathrm{K}}-\mathrm{U}\right)^{\mathrm{T}}$ and $\mathrm{S}_{\mathrm{w}}^{2 \mathrm{~d}}=$ $\frac{1}{\mathrm{~N}} \sum_{\mathrm{K}=1}^{\mathrm{L}} \sum_{\mathrm{i}=1}^{\mathrm{N}_{\mathrm{K}}}\left(\mathrm{X}_{\mathrm{i}}^{\mathrm{K}}-\mathrm{U}_{\mathrm{k}}\right)\left(\mathrm{X}_{\mathrm{i}}^{\mathrm{K}}-\mathrm{U}_{\mathrm{K}}\right)^{\mathrm{T}} \quad$ are $\quad$ between-class scatter matrix and within-class scatter matrix respectively.

\subsection{Feed-Forward Back-Propagation Neural Network}

The back-propagation neural network (BPNN) is the best known and widely used learning algorithm in training multilayer perceptron (MLP) [23]. Back propagation is a multi-layer feed forward, supervised learning network based on gradient descent learning rule. This BPNN provides a computationally efficient method for changing the weights in feed forward network, with differentiable activation function units, to learn a training set of inputoutput data[23].

A typical back propagation network [24] with multi-layer, feed-forward supervised learning is shown in Fig. 4. Here learning process in back-propagation requires pairs of input $\left(\mathrm{x}_{1}, \mathrm{x}_{2}\right.$, etc. $)$ and target vectors. The output vector ' $\mathrm{o}$ ' is compared with target vector 't '. In case of difference of ' $\mathrm{o}$ ' and ' $\mathrm{t}$ ' vectors, the weights are adjusted to minimize the difference. Initially random weights and thresholds are assigned to the network. These weights are updated every iteration in order to minimize the mean square error between the output vector and the target vector [23].

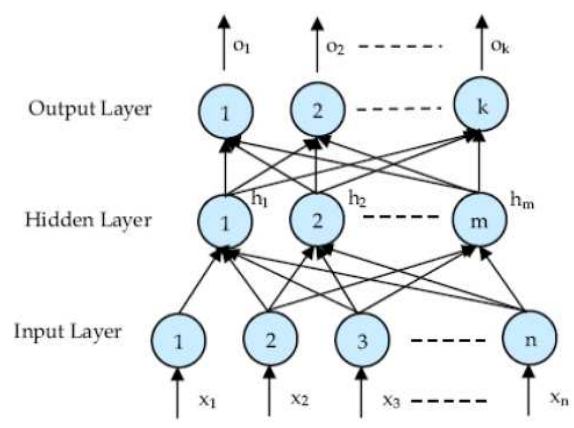

Fig 4. Basic block of Back-propagation neural networks.

Appropriate selection of the parameters used for training to ensure efficient operation. The initial weight will influence whether the net reaches a global or local minima of the error and if so how rapidly it converges. To get the best result the initial weights are set to random numbers between -1 and $1[23,24]$.

Training a net is performed in order to achieve a balance between memorization and generalization. It is not necessarily advantageous to continue training until the error reaches a minimum value. The weight adjustments are based on the training patterns. As long as the error for validation decreases training continues. Whenever the error begins to increase, the net is starting to memorize the training patterns. At this point training is terminated. If the activation function can vary with the function, then it can be seen that an input, $m$ output function requires at most $2 \mathrm{n}+1$ hidden units.

\subsection{Palmprint Database}

Hyperspectral palmprints database which is developed by the Biometric Research Centre at Department of Computing, the Hong Kong Polytechnic University has been used [25]. Hyperspectral palmprint images were collected from 190 volunteers. The age distribution is from 20 to 60 years old. The samples have been collected in two separate sessions. In each session, the subject was asked to provide around 7 images for each palmprint for each wavelength and the size for each palmprint is $128 \times 128$ pixels. Zhenhua, et.al [26] suggested using spectral band at $700 \mathrm{~nm}$ because it contains more discriminative information; thus, it is used in our experimental phase.

\section{Features Extraction and Recognition Results}

\subsection{Feature Extraction}

Low resolution palmprint images can be represented by some line features. The principle lines can be extracted using stack filters. However, these principal lines are not sufficient to represent the uniqueness of each individual's palmprint because different people may have similar 
principal lines in their palmprints; moreover, some palmprint images do not have clear wrinkles. Several techniques have been implemented to extract features from palmprint image such as wavelets, ridgelets, and other but these methods are unable to detect smooth edges which result from the conjunctions of principal lines and wrinkles. In the following the wavelets, ridgelets, curvelets and contourlets features have been extracted. These features have been projected in order to reduce the dimensionality by using 2D LDA. Finally, a vector which is a projection resultant is passed to the feed-forward neural network for training and testing phases.

$2 D$ Wavelet features; applying wavelet transformation leads to different band of wavelet coefficient of the original palmprint images. While high frequency components contribute to details, low frequency components contribute to approximation (general form of palmprint) image. A large variation of palmprint image is present in high frequency components and small effect in low frequency component. Each level of wavelet decomposition divides original palmprint image into four subbands leading to multiresolution analysis. Each subband can be used to extract feature. In this paper, $2^{\text {nd }}$ level of $2 \mathrm{D}$ discreet wavelet is applied, Fig. 6 shows the vertical coefficients of 1 st $2 \mathrm{D}$ discreet wavelet decomposition level which is taken as a palmprint features. 2DLDA has been applied to vertical coefficients leading to single vector which is passed to feed- forward back-propagation neural network for training or testing. Fig. 5 shows region of interest (ROI) which extracted from original palmprint image.

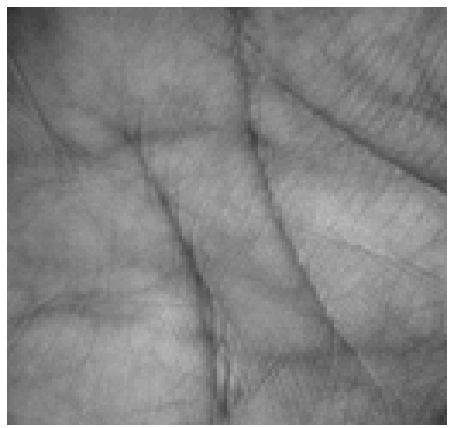

Fig 5. ROI of Palmprint

The unique coefficients which are used as discriminated features are obtained in this work from vertical coefficients only.

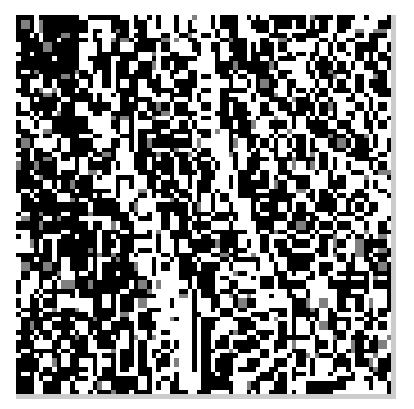

Fig 6. Vertical Coefficients
Ridgelet features; Ridgelet transform offers a mathematical framework in order to organize the liner information at different scale and resolution. First, the ridgelet transform is applied to palmprint images in order to convert them into time-frequency domain leading to ridgelet coefficients. Fig. 7 shows ridgelet transform features of palmprint image which was illustrated in Fig. 5.

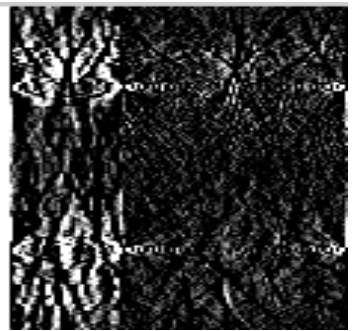

Fig7. Ridgelet features

When the palmprints are transformed by ridgelet transform, 2D LDA applied in order to reduce the dimensionally in order to obtain a projection resultant vector. Then, the resulted vector is passed to feed-forward back-propagations neural network for recognition phase.

Curvelet features; the combinations between 2D wavelets transform and ridgelets transform leads to curvelet transform. Each curvelet decomposition level leads to high number of curvelet coefficients. The most discriminative features can be obtained as shown in Fig. 8.

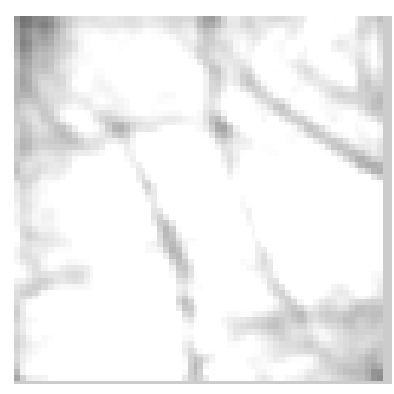

Fig 8. Curvelet Coefficients

Curvelab 2.1.2 [27], a Matlab toolbox includes the mirror extended curvelets has been used to extract curvelet coefficients. 2D LDA has been used to reduce the dimensionality in order to pass the most discriminative features to the back-propagations neural networks

Contourlets Features; the contourlet transform addresses the features extraction by providing two additional properties, directionality and anisotropy which provide contourlets transform with more efficient representation of the object.

Contourlet transform is applied to palmprint image using two different steps: first, a Laplacian pyramid is applied to decompose the image into two subbands or (cells) the first one is low pass filter and other is high pass filter for edge detection. Second a directional filter bank (DFB) is applied. The most discriminative contourlets coefficients which related to palmprint image in Fig. 5 appear in Fig. 9. 


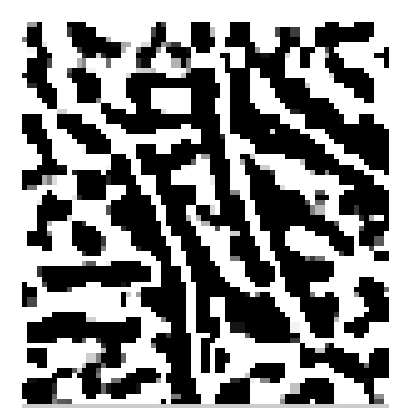

Fig 9. Contourlet features

The contourlets coefficients are reduced using 2D LDA in order to get a resulted discriminate vector to be passed into feed-forward back propagation neural network classifier.

\subsection{Recognition Results}

Table 1. Recognition Results

\begin{tabular}{ccc}
\hline Multiscale Transform & $\begin{array}{c}\text { Dimensionality } \\
\text { reduction 2D PCA }\end{array}$ & $\begin{array}{c}\text { Dimensionality } \\
\text { reduction 2D LDA }\end{array}$ \\
\hline 2D discrete wavelet & $87.5 \%$ & $93.3 \%$ \\
Ridgelet & $91.3 \%$ & $95.8 \%$ \\
Curvelet & $97.5 \%$ & $98.3 \%$ \\
Contourlet & $95.83 \%$ & $99.1 \%$ \\
\hline
\end{tabular}

A test samples of 30 persons have been taken into account with a total of 360 palmprint images divided as: 240 palmprints for training phase and 120 palmprints for testing phase. The palmprint images were transformed by using multiscale image transforms: 2D discrete wavelets, Ridgelet, Curvelet and Contourlet in order to extract the discriminative features for individual's. 2D LDA was used for projection in order to reduce the features size and to get a vector which contains the most discriminative features. The resulted vector is used as input to a feed forward backpropagation neural network classifier.

The higher recognition accuracy in Table 1 is obtained using contourlet transform, $99.1 \%$. The major attributes in the transformed palmprint image are principal line length and width, and wrinkles length and width. 2D LDA tries to identify attributes that account for the most variance between classes; thus, the 2D LDA is a supervised method, using known class labels. The class labels field is also called target field. By comparing recognition accuracy when it considered as a function of dimensionality reduction technique as shown in Table $12 \mathrm{D}$ LDA is outperformed previous results obtained using 2D PCA [28] over all multiscale transformation.

The recognition phase in this work has been divided into two stages; the first one is called training stage. Each feature vector which resulted from multiscale transformation and projected by 2D LDA is passed to feedforward back propagation neural network and trained using gradient function. The same transformation has been applied to palmprint images which are used in test stage but the resultant feature vector didn't trained. The learning rate was 0.05 .

The recognition accuracy for each multiscale transforms illustrated in Table 1. By comparing our work with similar works, we can see: In [4] the accuracy was $99.9 \%$. The dependency on highly energy components was insufficient to select the most discriminative feature and the whole recognition algorithm was time consuming and unreliable. The contourlet transform, PCA, and minimum distance classifier were used for transformation in [5] and the accuracy was $94 \%$ but the limitations in the classifier and database made the result inconsistent. In [6] the combination between contourlet and sub-sampled contourlet was used where Euclidean distance classifier was used. Different levels of accuracy were achieved but the classifier type, image size, and comparison were inadequate. In [7] wavelets and 2D PCA and the recognition accuracy was $97 \%$ but different limitations appear in this work such as the sample size was inconsistent, comparison with 1D projection techniques and 10 projection vectors were used in recognition, but our experimental work used only one projection vector.

\section{Conclusion}

This paper proposed a novel recognition approach based on their palmprints. The novelty of the approach is in the combinations of images transform techniques, 2D LDA features reduction technique and feed- forward NN classifier. PolyU pre-processed hyperspectral database was used and the recognition accuracy were 93.3\%, 95.8\%, $98.3 \%$, and $99.1 \%$ when 2D LDA was used to reduce the vector dimensions and: 2D discrete wavelet, ridgelet, curvelet, and contourlet applied respectively. The best result was obtained using contourlet.

For future work, the combinations between another multiscale image transformations such as shaplet, bandlet, platlet and other will be taken in addition to use another features reduction techniques such as independent component analysis (2D ICA), kernel PCA and other modern techniques are suggested to be used.

\section{References}

[1] D. Zhang, Wai-Kin Kong, J. You and Michael Wong, "Online palmprint identification," IEEE Transactions on Pattern Analysis and Machine Intelligence, vol. 25, pp. 1041-1050, Sept. 2003.

[2] A. Jain, R. Bolle and S. Pankanti (eds.), Biometrics: Personal Identification in Networked Society, Boston, Mass: Kluwer Academic Publishers, 1999.

[3] A. Kong, D. Zhang and M. Kamel, "A survey of palmprint recognition," Journal of Pattern Recognition, vol. 42, pp. 1408-1418, July. 2009. 
[4] H.B.Kekre, R. Vig and S. Bisani, "Identification of multispectral palmprints using energy compaction by hybrid wavelet," International Conference on Biometric (ICB), pp. 433-438, March. 2012.

[5] M. Sharkas, I. El-Rube and M.A. Mostafa, "The contourlet transform with the principal component analysis for palmprint recognition," International Conference on Computational Intelligence, Communication Systems and Networks (CICSyN), pp. 262-267, July. 2010.

[6] H.Masood, M. Asim, M. Mumtaz and A. Mansoor,'Combined contourlet and non-subsampled contourlet transforms based approach for personal identification using palmprint," Digital Image Computing: Techniques and Applications, DICTA '09, pp.408-415, Dec.2009.

[7] J. Andrew Bangham and Richard V. Aldridge, "Multiscale decomposition using median and morphological filters," IEEE Winter Workshop on Nonlinear Digital Signal Processing, 6.1 1.1 - 6.1_1.4, 1993.

[8] Alexandru Isar, Sorin Moga, and Xavier Lurton, "A statistical analysis of the $2 \mathrm{~d}$ discrete wavelet transform," Proceedings of the International Conference AMSDA 2005, 1275-1281, 17-20 May 2005

[9] Samuel Foucher, Goz'e Bertin B'eni'e, Jean-Marc Boucher,"Multiscale map filtering of sar images," IEEE Transactions on Image Processing, vol. 10, no.1, January 2001, 49-60.

[10] E. J. Cand $\mu$ es, Ridgelets: Theory and Applications, Ph.D. thesis, Department of Statistics, Stanford University, 1998.

[11] E. J. Candues and D. L. Donoho, "Ridgelets: a key to higher- dimensional intermittency?" Phil. Trans. R. Soc. Lond. A., pp. 2495-2509, 1999.

[12] G. T. Herman, Image Reconstruction from Projections: The Fundamentals of Computerized Tomography, Academic Press, 1980.

[13] A. Rosenfeld and A. C. Kak, Digital Picture Processing, Aca- demic Press, 2nd edition, 1982.

[14] H. Führ, L. Demaret and F. Friedrich, Beyond wavelets: new image representation paradigms. Book chapter. In: M. Barni and F. Bartolini (Eds.), Document and Image Compression, CRC Press, Boca Raton, FL, 2006.

[15] E. Candes and D. Donoho. "New tight frames of curvelets and optimal representations of objects with C2 singularities," Commun. Pure Appl. Math. 57, pp. 219-266, 2004.

[16] J. Starck, E. Candes and D. Donoho. "The Curvelet
Transform for Image Denoising," IEEE Transactions on Image Processing, 11, pp. 670-684, 2002.

[17] M. Do. Directional multiresolution image representation, Thesis, Swiss Federal Institute of Technology, 2001.

[18] M. Do and M. Vetterli. "The contourlet transform: an efficient directional multiresolution image representation," IEEE Transactions Image on Processing, 14 (12), pp. 20912106, Dec. 2005

[19] R. Bamberger and M. Smith. "A filter bank for the directional decomposition of images: Theory and design," IEEE Trans. Signal Proc. 40, 1992, pp. 882-893.

[20] Th Liwei Wang, Xiao Wang, Xuerong Zhang and Jufu Feng, "The equivalence of two-dimensional PCA to line-based PCA," Pattern Recognition Letters, 26 (1), pp. 57-60, Jan 2005.

[21] J. Yang and D. Zhang,"Two-dimensional PCA: A new approach to appearance-based face representation and recognition," IEEE Trans. Pattern Anal. Machine Intell. PAMI-26 (1), 131-137, 2004

[22] W.S. Zheng, J.H. Lai, S.Z. Li, "1D-LDA vs. 2DLDA: When is vector-based linear discriminate analysis better than matrix-based?" Pattern Recognition, vol. 41, pp. 2156-2172, July 2008.

[23] P.Latha, L.Ganesan and S.Annadurai, "Face recognition using neural networks," Signal Processing: An International Journal (SPIJ) 3 (5), 153-160, Nov 2009.

[24] S.Lawrence, C.L.Giles, A.C.Tsoi, and A.d.Back, "Face recognition: a convolutional neural network approach," IEEE Transactions of Neural Networks, vol.8, no.1, pp.98113, 1993.

[25] Department of Computing, The Hong Kong Polytechnic University (PolyU), Hyperspectra Palmprint database, Polyu, accessed on Aug. 22, 2013, available at: http://www4.comp.polyu.edu.hk/ biometrics/Hyperspectral Palmprint/HSP.htm.

[26] Zhenhua Guo, Lei Zhang, and David Zhang, "feature band selection for multispectral palmprint recognition," International Conference on Pattern Recognition, pp. 11361139, 2010.

[27] The Curvelet.org. Accessed: September, 2013. Available at: http://www.curvelet.org/

[28] H. Elaydi, M. Alhanjouri, and M. Abukmeil, "Palmprint recognition using 2-d wavelet, ridgelet, curvelet and contourlet," to appear in i-manager's Journal on Electrical Engineering (JEE), 2013. 